\title{
Smoluchowski equation approach for quantum Brownian motion in a tilted periodic potential
}

\author{
William T. Coffey, ${ }^{1}$ Yuri P. Kalmykov, ${ }^{2}$ Serguey V. Titov, ${ }^{3}$ and Liam Cleary ${ }^{1}$ \\ ${ }^{1}$ Department of Electronic and Electrical Engineering, Trinity College, Dublin 2, Ireland \\ ${ }^{2}$ Laboratoire de Mathématiques, Physique et Systèmes, Université de Perpignan, 52, Avenue de Paul Alduy, 66860 Perpignan Cedex, \\ France \\ ${ }^{3}$ Institute of Radio Engineering and Electronics, Russian Academy of Sciences, Vvedenskii Square 1, Fryazino, 141190, Russia
}

(Received 8 May 2008; published 10 September 2008)

\begin{abstract}
Quantum corrections to the noninertial Brownian motion of a particle in a one-dimensional tilted cosine periodic potential are treated in the high-temperature and weak bath-particle coupling limit by solving a quantum Smoluchowski equation for the time evolution of the distribution function in configuration space. The theoretical predictions from two different forms of the quantum Smoluchowski equation already proposedviz., J. Ankerhold et al. [Phys. Rev. Lett. 87, 086802 (2001)] and W. T. Coffey et al. [J. Phys. A 40, F91 (2007)] - are compared in detail in a particular application to the dynamics of a point Josephson junction. Various characteristics (stationary distribution, current-voltage characteristics, mean first passage time, linear ac response) are evaluated via continued fractions and finite integral representations in the manner customarily used for the classical Smoluchowski equation. The deviations from the classical behavior, discernible in the dc current-voltage characteristics as enhanced current for a given voltage and in the resonant peak in the impedance curve as an enhancement of the $Q$ factor, are, respectively, a manifestation of relatively high-temperature nondissipative tunneling (reducing the barrier height) and dissipative tunneling (reducing the damping of the Josephson oscillations) near the top of a barrier.
\end{abstract}

DOI: 10.1103/PhysRevE.78.031114

PACS number(s): 05.40.-a, 03.65.Yz, 05.60.Gg, 74.50.+r

\section{INTRODUCTION}

The Wigner representation [1] or phase-space formulation of quantum mechanics in terms of quasiprobability distributions of the canonical variables, also known as the Moyal quantization [2], allows quantum-mechanical expectation values involving the density matrix to be calculated just as classical ones and is eminently suited to the calculation of quantum corrections to these. The Wigner representation contains only features common to both quantum and classical statistical mechanics and formally represents quantum mechanics as a statistical theory on classical phase space. Wigner's phase space or, more generally, representation space formalism, originally developed [1] for closed quantum systems in order to obtain quantum corrections to classical thermodynamic equilibrium, is useful in diverse branches of physics (see, e.g., [3-7]). In particular, it may also be applied to open quantum systems [8], providing a useful tool for the calculation of quantum corrections to classical models of dissipation such as Brownian motion (see, for example, [9-14]). In this context the one-dimensional quantum Brownian motion of a particle of mass $m$ moving in a potential $V(x)$ is usually studied by regarding the Brownian particle as bilinearly coupled to a bath of harmonic oscillators in thermal equilibrium at temperature $T$. The dynamics of the particle are described by a master equation for the time evolution of the Wigner distribution $W(x, p, t)$ in the phase space $(x, p)$ of positions and momenta of the particle. This equation is a partial differential equation in phase space akin to the classical Fokker-Planck equation so that operators are not involved. By using existing powerful computational techniques developed for the Fokker-Planck equation [15], quantum effects on diffusive transport properties can then, in principle, be estimated for arbitrary potentials and in a wide range of dissipation parameters (see, e.g., [16-18]).
The description of the quantum dynamics of a Brownian particle can be considerably simplified in the limit of very high dissipation (VHD) to the heat bath (i.e., the noninertial limit) just as in the classical theory of Brownian motion, where the underlying kinetic equation is the Smoluchowski equation for the configuration-space probability distribution function $P(x, t)$. As shown recently $[19,20]$, the phase-space formalism can be used to derive a quantum Smoluchowski equation (QSE) for the configuration-space probability distribution function for noninertial translational Brownian motion via perturbation theory in $\hbar^{2}$ ( $\hbar$ is Planck's constant). The QSE obtained in $[19,20]$ governs the time evolution of the quasiprobability density in configuration space and characterizes the motion of a quantum Brownian particle in the VHD limit. It is derived [19] by first writing a master equation for the time evolution of the Wigner distribution $W(x, p, t)$ in the phase space $(x, p)$ of positions and momenta of the particle $[11-13,20]$ : namely,

$$
\frac{\partial W}{\partial t}+\frac{p}{m} \frac{\partial W}{\partial x}-\frac{1}{i \hbar}\left[V\left(x+\frac{i \hbar}{2} \frac{\partial}{\partial p}\right)-V\left(x-\frac{i \hbar}{2} \frac{\partial}{\partial p}\right)\right] W=\hat{M}_{D} W,
$$

where $\hat{M}_{D}$ is the collision kernel operator. The left-hand side of this equation is the quantum analog of the classical Liouville equation pertaining to a closed system, where the collision term is zero. This situation was first discussed in the context of quantum corrections to classical thermodynamic equilibrium by Wigner [1]. He solved the closed (equilibrium) equation for relatively high temperatures using perturbation expansions in Planck's constant, thus yielding the Wigner stationary distribution $W_{0}(x, p)$. The ultimate objective of his investigation [21] was to obtain quantum corrections (due to high-temperature tunneling near the top of the 
potential barrier) to classical reaction rate or transition state theory (TST). This theory is based on the assumption that thermodynamic equilibrium prevails everywhere in the relevant potential well so that the closed equation applies; i.e., the Brownian motion is ignored. The Wigner results, which take the form of the classical TST rate $\Gamma_{T S T}^{c l}$ multiplied by a temperature- and potential-dependent quantum correction factor $\Xi$ causing an effective lowering of the potential barrier (thus increasing the reaction rate), are limited to relatively high temperatures. This is so because in calculating the quantum-corrected reaction rate, the potential is replaced by that of a harmonic oscillator near the bottom of a well, while near the top it is replaced by that of an inverted harmonic oscillator [21]. Returning to the nonequilibrium situation under discussion (where the Brownian motion is included by representing the collision kernel as essentially a KramersMoyal expansion truncated at the second term [19,20]), the right-hand side of the master equation (1) - i.e., the collision kernel operator $\hat{M}_{D}$-describes the bath-particle interaction, pertaining to an open system. Hence, assuming frequencyindependent damping [13,22] (which is valid for a wide range of parameters in both weak and strong damping limits), the diffusion coefficients appearing in the operator $\hat{M}_{D}$ may be calculated to any order of perturbation theory in $\hbar^{2}$ in a manner analogous to Wigner's perturbation solution of the closed equation. This is accomplished by postulating the Wigner stationary distribution $W_{0}(x, p)$ as the equilibrium solution of the master equation (1). The imposition of the Wigner stationary distribution as the equilibrium distribution in order to calculate diffusion coefficients has been successfully applied in the quantum Brownian motion of particles $[17,18]$ and spins [23]. For point particles with separable and additive Hamiltonians, the procedure is exactly analogous to the ansatz, in classical Brownian motion, that the MaxwellBoltzmann distribution is the stationary solution of the underlying Fokker-Planck (in this particular context called the Klein-Kramers) equation for the phase-space evolution of the joint distribution of positions and momenta. For spins, which have no classical analog and, in general, nonseparable Hamiltonians, the representation space [24] is the space of polar and azimuthal angles $(\vartheta, \varphi)$ constituting the canonical variables. Thus, the classical equilibrium distribution, assumed in order to calculate diffusion coefficients in the Fokker-Planck equation for the distribution of spin orientations, is the Boltzmann distribution of orientations. In the quantum spin case, however, the stationary Wigner distribution must be determined from first principles for each particular case of the spin Hamiltonian operator [23,24].

Returning to particles, having determined the phase-space diffusion coefficients, integration of the phase-space master equation (1) over the momenta, and proceeding to the VHD limit $[18,19]$ exactly as for the classical Smoluchowski equation [25-28], then leads to the QSE for the quasiprobability density function $P(x, t)$ in configuration space $(x)$ : namely,

$$
\frac{\partial P(x, t)}{\partial t}=\frac{\partial}{\partial x}\left\{\frac{P(x, t)}{\zeta} \frac{\partial V(x)}{\partial x}+\frac{\partial}{\partial x}[D(x) P(x, t)]\right\} .
$$

Here

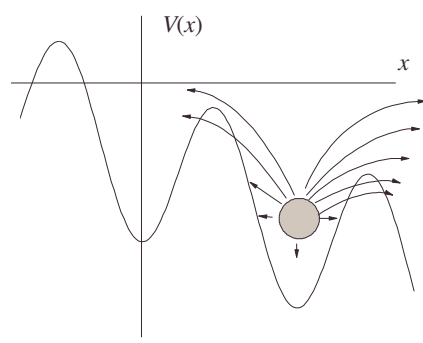

FIG. 1. (Color online) Overdamped quantum Brownian motion of a particle in a tilted periodic potential: the particle may escape from a potential well both over (due to thermal agitation) and below (due to tunneling through) the higher and lower potential barriers.

$$
\begin{aligned}
D(x)= & \frac{1}{\zeta \beta}\left(1+2 \Lambda V^{\prime \prime}(x)-\frac{4 \Lambda^{2}}{5}\left\{\left[V^{\prime \prime}(x)\right]^{2}+3 V^{\prime}(x) V^{(3)}(x)\right.\right. \\
& \left.\left.-3 \beta^{-1} V^{(4)}(x)\right\}+\cdots\right)
\end{aligned}
$$

is the quantum diffusion coefficient, $\Lambda=\hbar^{2} \beta^{2} /(24 m)$ is the characteristic quantum parameter, $\beta=(k T)^{-1}, k$ is Boltzmann's constant, $T$ is the temperature, $m$ is the mass of the Brownian particle, $\zeta=\gamma m$ is the friction coefficient, where $\gamma$ is a dissipation (damping) parameter characterizing the bathparticle interaction, and of course $(\zeta \beta)^{-1}$ is the classical diffusion coefficient determined by Einstein. The drift coefficient $-\zeta^{-1} \partial_{x} V$ in Eq. (2), on the other hand, coincides with its classical counterpart. The quasiprobability density $P(x, t)$ is simply the trace of the density matrix operator $\hat{\rho}$-namely, $\langle x|\hat{\rho}| x\rangle$-constituting an example of Weyl's correspondence [29] between quantum-mechanical operators on Hilbert space and ordinary $c$-number functions in phase space. The Weyl correspondence inter alia allows averages of quantummechanical operators to be calculated just as classical averages using the Weyl symbol of the operator. Now the QSE resembles the classical Smoluchowski equation; however, unlike that equation, the diffusion coefficient $D(x)$ depends on the derivatives of the potential and the characteristic quantum parameter $\Lambda$, while the drift coefficient remains the same. [Equation (2) reduces to the classical Smoluchowski equation for the configuration-space distribution function $P(x, t)$ when the quantum parameter $\Lambda=0]$. Just as the classical case, the QSE describes the long-time (or lowfrequency) relaxation behavior of a system $[15,28]$.

Here we solve the QSE (2) for the particular case of a tilted cosine (or biased washboard) potential in order to evaluate quantum corrections (at relatively high temperatures) to various characteristics to first and second order of perturbation theory in $\hbar^{2}$. The model of Brownian motion of a particle in a tilted cosine potential (see Fig. 1) arises in a number of important physical applications. We mention Josephson junctions [30-32], ring-laser gyroscopes [33], the dynamics of a charged-density-wave condensate in an electric field [34], etc., and many other processes involving quantum and classical Brownian motion in periodic structures [35]. As a particular example of an application of the QSE, we shall now estimate quantum effects in the dynamics of a point Josephson junction. In the context of classical 
Brownian motion, this system has been described in detail in Refs. [30-32] (see also [28], Chap. 5). The dc current voltage characteristics of a Josephson junction in the classical Smoluchowski regime were derived by Ambegaokar and Halperin [36] and by Ivanchenko and Zil'berman [37]. Various aspects of quantum effects in the characteristics of Josephson junctions have already been analyzed, e.g., in Refs. [38-42]. Here, we shall include quantum effects in the stationary distribution, the dc current-voltage characteristic, the normalized differential resistance, and the nonstationary problem of the linear impedance of a point Josephson junction (ignoring the capacitance, which corresponds to the noninertial or very high damping limit) by solving the QSE using the continued-fraction methods already developed for the classical problem $[15,28]$. Moreover, we shall demonstrate that the effective eigenvalue method [28] yields closed analytic solutions for the junction impedance as two Lorentzians just as in the classical problem [28]. These have a simple physical explanation as a damped resonant circuit with the decay rate and resonant frequency of the oscillations given by the real and imaginary parts of the effective eigenvalue. We shall also calculate the mean first passage time for a particle to leave a well of the potential. The mean first passage time may be determined, just as the classical case, knowing the quantum diffusion coefficient and stationary distribution only [15]. Moreover, the convergence of the perturbation procedure in $\hbar^{2}$ will be tested by comparing the first and second order of perturbation theory solutions. We remark that a feature of the present problem is that the firstorder perturbation solution of the QSE reduces to a threeterm scalar recurrence relation, just as the corresponding classical problem [15]. This relation may then be solved using scalar continued fractions. If the calculation is carried to the second order of perturbation theory, however, one must always resort to matrix continued fractions, as a five-term recurrence relation is now involved. Nevertheless, the matrix continued fraction so generated is much simpler than that obtained [17] using the master equation (which is valid for all dissipation regimes) for the phase space distribution $W(x, p, t)$. Yet another simplification stemming from the QSE is that the diffusion coefficient may be calculated to any order of perturbation theory from the equilibrium Wigner configuration-space distribution $P_{W}(x)$ without recourse to the complete phase-space master equation as we shall presently demonstrate. This is accomplished following the original method of Einstein and Smoluchowski [28] for the noninertial classical Brownian motion by using the explicit form of the equilibrium solution of the QSE. All the foregoing results will then be compared with those calculated from the QSE recently proposed by Ankerhold et al. [43] from the path-integral representation of dissipative quantum mechanics. This equation has already been used in many applications of the quantum Brownian motion in a periodic potential (see, e.g., $[41,42,44-47])$.

\section{DERIVATION OF THE QSE}

The dynamics of a quantum system described by the semiclassical Smoluchowski equation (2) may be equiva- lently described using a quantum analog of the Langevin equation with multiplicative noise. The Langevin equation corresponding to Eq. (2) in the Stratonovich interpretation $[15,28]$ reads

$$
\dot{x}(t)=-\frac{1}{\zeta} \partial_{x}\left\{V[x(t)]+\frac{\zeta}{2} D[x(t)]\right\}+\sqrt{\frac{\beta}{\zeta} D[x(t)] \lambda}(t),
$$

where the diffusion coefficient $D(x)$ from Eq. (3) depends on the derivatives of the potential and the characteristic quantum perturbation parameter $\Lambda=\hbar^{2} \beta^{2} /(24 m)$, the overdot denotes the time derivative, and $\lambda(t)$ is a random force with the Gaussian white noise properties

$$
\overline{\lambda(t)}=0, \overline{\lambda(t) \lambda\left(t^{\prime}\right)}=(2 \zeta / \beta) \delta\left(t-t^{\prime}\right)
$$

(the overbar means the statistical average over the realizations of the random force). Equation (4) may be used as an approximate description of the kinetics of a quantum Brownian particle in the VHD (or noninertial) limit. By averaging Eq. (4), one obtains the averaged equation of motion

$$
\overline{\dot{x}}=-\zeta^{-1} \overline{\partial_{x} V(x)}
$$

and thus the drift coefficient, coinciding with its classical counterpart.

The explicit form of the diffusion coefficient $D(x)$ in Eq. (3) has been obtained in [19,20] from the high-damping (noninertial) limit of the phase-space master equation (1). As we have briefly mentioned above, yet another simpler method of determining $D(x)$, avoiding the time-dependent phase-space master equation entirely, is based on an extension of the ansatz that the Boltzmann distribution is the equilibrium solution of the classical Smoluchowski equation. This simple heuristic idea was originally used by Einstein, Smoluchowski, and others [28] in order to calculate the drift and diffusion coefficients. By extending this idea, the drift and diffusion coefficients can be directly evaluated in the QSE from a knowledge of the equilibrium configurationspace quantum distribution only. In order to determine the explicit form of the diffusion coefficient $D(x)$ in Eq. (3), we first recall that the stationary distribution function in configuration space, $P_{W}(x)$, must be a stationary solution of the QSE (2). Here the simplification occurs because $P_{W}(x)$ can be evaluated directly from the stationary Wigner distribution function $W_{0}(x, p)$ in the phase space of coordinates and momenta $(x, p)$ since by definition $[1,20]$

$$
P_{W}(x)=\int_{-\infty}^{\infty} W_{0}(x, p) d p .
$$

Now the Wigner distribution can in general be developed in a power series in $\hbar^{2}$ as [1]

$$
W_{0}(x, p)=e^{-\beta \varepsilon(x, p)}+\hbar^{2} w_{2}(x, p)+\hbar^{4} w_{4}(x, p)+\cdots,
$$

where $\varepsilon(x, p)=p^{2} / 2 m+V(x)$ is the classical energy. Furthermore, the perturbed functions $w_{2 r}(x, p)$ can be evaluated explicitly in terms of the derivatives of $V(x)$ to any desired power of $r$ as described by Wigner [1], where explicit equations for $w_{2}(x, p)$ and $w_{4}(x, p)$ are given. The stationary dis- 
tribution function in configuration space, $P_{W}(x)$, can then be evaluated to any desired power of $r$ by simply integrating $W_{0}(x, p)$ with respect to the momentum $p$. The explicit equation for $P_{W}(x)$ to second order in $\hbar^{2}$ is [20]

$$
\begin{aligned}
P_{W}(x)= & e^{-\beta V}\left\{1+\Lambda\left[\beta\left(V^{\prime}\right)^{2}-2 V^{\prime \prime}\right]+\frac{\Lambda^{2}}{10}\left[36 V^{\prime \prime 2}+48 V^{\prime \prime \prime} V^{\prime}\right.\right. \\
& \left.\left.-44 \beta V^{\prime \prime} V^{\prime 2}+5 \beta^{2} V^{\prime 4}-24 V^{(4)} / \beta\right]+\cdots\right\} .
\end{aligned}
$$

Now, according to our ansatz, the stationary distribution $P_{W}(x)$ must be the stationary solution of the postulated timedependent equation (2); i.e., it satisfies

$$
\frac{\partial}{\partial x}\left\{\frac{P_{W}}{\zeta} \frac{\partial V}{\partial x}+\frac{\partial}{\partial x}\left(D P_{W}\right)\right\}=0 .
$$

Hence, seeking the diffusion coefficient $D(x)$ as a power series in $\hbar^{2}$, viz.,

$$
D(x)=(\zeta \beta)^{-1}+\hbar^{2} d_{2}(x)+\hbar^{4} d_{4}(x)+\cdots,
$$

one finds [substituting Eq. (8) into Eq. (7)] the spatially dependent $D(x)$ given by Eq. (3). Thus the imposition of the Wigner configuration-space distribution $P_{W}(x)$ as the equilibrium solution of Eq. (2), yielding a diffusion coefficient $D$ depending on the derivatives of the potential, is the exact analog of the ansatz of a Boltzmann stationary solution in the classical theory.

We remark that in the derivation of $D(x)$ above, we have imposed $P_{W}(x)$ as the stationary solution of the QSE (2), as determined from the stationary Wigner phase-space distribution function $W_{0}(x, p)$ in the approximation of frequencyindependent damping. In the high-temperature limit, viz. $[8,19,20]$,

$$
T \gg T_{0}=\hbar \zeta /(2 \pi m k),
$$

this approximation may be used in a wide range of model parameters if the interactions between the Brownian particle and the heat bath are small enough to allow one to use the weak-coupling limit and if the correlation time characterizing the bath is so short that we can regard the stochastic process originating in the bath as Markovian (a detailed discussion of the validity of this approximation is given by Weiss [8] and Grabert [48]). For parameter ranges, where such an approximation is invalid (e.g., throughout the verylow-temperature region, where non-Markovian effects are substantial), other methods should be used. We have also chosen such an approximation because our present objective is to understand how quantum effects treated in semiclassical fashion alter the classical Brownian motion in a potential in the Smoluchowski (VHD) regime where frequencyindependent damping is always assumed. Now the distribution $W_{0}(x, p)$ describes the system in thermal equilibrium without coupling to the thermal bath and corresponds to the canonical density matrix $\hat{\rho}_{e q}=e^{\beta \hat{H}} / Z(\hat{H}$ is the Hamiltonian of the system and $Z$ is the partition function) [1]; i.e., it pertains to the closed system. The ansatz that the equilibrium distribution corresponds to the canonical distribution has been successfully used both by Gross and Lebowitz [49] in their formulation of quantum kinetic models of impulsive collisions and by Redfield [50] in the calculation of the matrix elements of the relaxation operator in the context of his theory of relaxation processes. However, by the theory of quantum open systems [8], the equilibrium state in general may deviate from the canonical distribution $\hat{\rho}_{e q}$, insofar as the canonical distribution describes the thermal equilibrium of the system in the weak-coupling and high-temperature limits only. A detailed discussion of this problem is given, e.g., by Geva et al. [51]. Thus, in general in open quantum systems, the equilibrium phase-space distribution may depend on the damping and hence deviate from the canonical distribution $W_{0}(x, p)$. However, one may in general conclude that the damping-independent stationary distribution will reproduce the correct quantum results if the condition embodied in Eq. (9) is satisfied.

Now, the QSE deduced by Ankerhold et al. [43] is very similar but not identical to Eq. (2). In the high-temperature limit, that equation reads (in our notation)

$$
\frac{\partial P}{\partial t}=\frac{\partial}{\partial x}\left\{\frac{P}{\zeta} \frac{\partial}{\partial x} V_{e f}+\frac{\partial}{\partial x}[D P]\right\},
$$

where $V_{e f}=V(x)+\Lambda V^{\prime \prime}(x) / \beta$ is the effective potential. We see that Eq. (10) differs from Eq. (2) by the additional term in $V_{\text {eff }}$. However, this difference is important, because the stationary solution of Eq. (10) is

$$
P_{A}(x) \sim e^{-\beta V(x)}\left\{1+\Lambda\left[\beta V^{\prime}(x)^{2}-3 V^{\prime \prime}(x)\right]+\cdots\right\} .
$$

The true Wigner equilibrium distribution in configuration space, $P_{W}(x)$, from Eq. (6) does not, however, coincide with the stationary distribution $P_{A}(x)$ and so does not satisfy Eq. (10).

\section{APPLICATION TO A TILTED PERIODIC POTENTIAL}

As a particular example of the solution of Eq. (2), we consider the one-dimensional translational Brownian motion of a particle in a tilted periodic potential:

$$
V(x)=-V_{0} \cos (2 \pi x / a)-F x,
$$

where $a$ is a characteristic length. On introducing the normalized coordinate $x$, time $\tau$, tilt $y$, barrier parameter $b$, and quantum parameter $\Lambda$ as

$$
\begin{gathered}
\beta V(x) \rightarrow V(x), \quad y=\frac{a F}{2 \pi V_{0}}, \quad \beta V_{0}=b, \quad \frac{2 \pi x}{a} \rightarrow x, \\
\frac{t}{\tau} \rightarrow t, \quad \tau=\frac{\zeta \beta a^{2}}{4 \pi^{2}}, \quad \Lambda \frac{8 \pi^{2}}{\beta a^{2}} \rightarrow \Lambda,
\end{gathered}
$$

the QSE (2), becomes

$$
\begin{aligned}
\frac{\partial P}{\partial t}= & \frac{\partial}{\partial x}\left\{V^{\prime} P+\frac{\partial}{\partial x}\right. \\
& \left.\times\left[1+\Lambda V^{\prime \prime}-\frac{\Lambda^{2}}{5}\left(V^{\prime \prime 2}+3 V^{\prime} V^{(3)}-3 V^{(4)}\right)+\cdots\right] P\right\},
\end{aligned}
$$

where the potential in dimensionless variables becomes 


$$
V(x)=-b(\cos x+y x) .
$$

In the application to a Josephson junction, modeled by an equivalent parallel electric circuit consisting of a resistance $R$ in parallel with a capacitance $C$ across which is connected an ideal current generator (representing the bias current $I_{d c}$ applied to the junction) [30-32], the mass $m$ and the friction coefficient $\zeta$ of the mechanical Brownian particle must be replaced by the corresponding electrical parameters $R$ and $C$ (resistance and capacitance of the junction) via

$$
m=C(\hbar / 2 e)^{2}, \quad \zeta=(\hbar / 2 e)^{2} / R,
$$

where $e$ is the charge of the electron. Here the parameter $b$ means the normalized (in the thermal energy $k T$ ) Josephson coupling energy and the tilt parameter $y$ is the ratio of the bias current $I_{d c}$ to the supercurrent amplitude $I$, while the dimensionless coordinate $x$ (meaning the phase difference between the wave functions for two superconductors) is given by the Josephson equation [30-32]

$$
\dot{x}(t)=2 e v(t) / \hbar,
$$

where $v(t)$ is the potential difference across the junction. The validity of the QSE (13) for the problem in question may be justified as follows. Noting that $\gamma=\zeta / m=1 /(R C)$, we can estimate $T_{0}$ on the right-hand side of Eq. (9) for typical values of $R$ and $C$ for Josephson junctions, as studied, for example, by Anderson and Goldman [52] and Falco et al. [53] (here the effects of thermal noise on current-voltage characteristics of Josephson junctions have been measured experimentally and have been compared with the model of Ambegaokar and Halperin [36] and Ivanchenko and Zil'berman [37]). For $R=1.3 \Omega$ and $C=245 \mathrm{pF}$ [52] and $R=0.2 \Omega$ and $C=1200 \mathrm{pF}$ [53], we have, respectively, $T_{0} \sim 0.004 \mathrm{~K}$ and $T_{0} \sim 0.005 \mathrm{~K}$. These estimations show that the values of $T_{0}$ for the two examples quoted above are very low in comparison with the temperatures of interest (which are also very low; for example, in both references experimental data were given in the temperature range $T \sim 1.4-4.2 \mathrm{~K}$ ). Thus the condition of applicability of our QSE, $T \gg T_{0}$, is perfectly fulfilled (at least as far as the two examples quoted are concerned). One can also estimate the Josephson plasma frequency $\omega_{0}=\sqrt{2 e I /(\hbar C)}$ for the given examples. This characteristic frequency determines the conditions of applicability of Ankerhold's QSE

$$
T \gg T_{A}=\frac{\hbar \omega_{0}^{2}}{2 \pi k \gamma} .
$$

From data given in Refs. [52,53], we have, respectively, $\omega_{0}$ $\sim 6 \times 10^{9} \mathrm{~s}^{-1}$ and $T_{A} \sim 0.01 \mathrm{~K}$ (i.e., $T_{A}>T_{0}$ ) and $\omega_{0} \sim 6$ $\times 10^{8} \mathrm{~s}^{-1}$ and $T_{A} \sim 0.00015 \mathrm{~K}$ (i.e., $T_{A}<T_{0}$ ). These estimations show that the temperature $T_{A}$ is also very low and can be both smaller and larger than $T_{0}$.

In many physical applications, a periodic solution $P(x, t)$ of Eq. (13) is required. This may be expanded in a Fourier series in $x$ : viz. $[15,28]$,

$$
P(x, t)=\frac{1}{2 \pi} \sum_{n=-\infty}^{\infty} c_{n}(t) e^{i n x} .
$$

By substituting Eq. (15) into Eq. (13), we find that the Fourier coefficients (statistical moments)

$$
c_{n}(t)=\left\langle e^{-i n x}\right\rangle(t)=\int_{0}^{2 \pi} e^{-i n x} P(x, t) d x
$$

satisfy the following differential-recurrence relation to second order in the perturbation parameter $\Lambda$ :

$$
\begin{aligned}
\frac{d}{d t} c_{n}(t)+\left(n^{2}+i n b y\right) c_{n}(t) & \\
= & \frac{b n}{2}\left[(1-n \Lambda) c_{n-1}(t)-(1+n \Lambda) c_{n+1}(t)\right] \\
+ & \frac{(b n \Lambda)^{2}}{5}\left[c_{n-2}(t)+\frac{3(1-i b y)}{2 b} c_{n-1}(t)-c_{n}(t)\right. \\
+ & \left.\frac{3(1+i b y)}{2 b} c_{n+1}(t)+c_{n+2}(t)\right] .
\end{aligned}
$$

This recurrence relation will yield the time-dependent periodic solution in the second order of perturbation theory. It is obvious that the first-order perturbation solution constitutes a three-term recurrence relation similar to that encountered in the classical case $[15,28]$.

In like manner, the periodic solution $P^{A}(x, t)$ of Eq. (10) can be expanded in a Fourier series in $x$. Thus substituting $P^{A}(x, t)=\sum_{n=-\infty}^{\infty} c_{n}^{A}(t) e^{i n x} / 2 \pi$ into Eq. (10), the corresponding Fourier coefficients $c_{n}^{A}(t)$ again satisfy a three-term differential recurrence relation to first order in $\Lambda$ : viz.,

$$
\begin{aligned}
\frac{d}{d t} c_{n}^{A}(t)+\left(n^{2}+\text { inby }\right) c_{n}^{A}(t)= & \frac{b n}{2}\left\{[1-(n+1 / 2) \Lambda] c_{n-1}^{A}(t)\right. \\
& \left.-[1+(n-1 / 2) \Lambda] c_{n+1}^{A}(t)\right\}
\end{aligned}
$$

In the classical limit $(\Lambda=0)$, both Eqs. (16) and (17) become the known differential-recurrence relation for the classical statistical moments [15,28]. Methods of solution of Eqs. (16) and (17) are described in detail in [15,28]. We shall first consider the stationary solution of Eq. (16) using these. To simplify the analysis and to facilitate the comparison with predictions of the QSE of Ankerhold et al. [43], Eq. (10), we may neglect the second-order correction term in Eq. (16) [so that just as the classical case, the differential-recurrence equation becomes a three-term recurrence relation] and present the solution to terms linear in the quantum correction factor $\Lambda$ : i.e., $o(\Lambda)$.

\section{STATIONARY PERIODIC SOLUTION OF THE QSE}

The periodic stationary solution $P_{s t}(x)$ of Eq. (13) can be obtained following the method used in [15] for the classical Smoluchowski equation from the equation for the probability current $S$ (which is constant in this case): 


$$
P_{s t}\left(\frac{\partial V}{\partial x}+\Lambda \frac{\partial^{3} V}{\partial x^{3}}\right)+\left(1+\Lambda \frac{\partial^{2} V}{\partial x^{2}}\right) \frac{\partial P_{s t}}{\partial x}=-S
$$

(for simplicity, we shall consider only terms linear in $\Lambda$ as is consistent with perturbation theory to first order in $\Lambda$ ). Solving Eq. (18) for $P_{s t}(x)$ and using the properties of the periodic solution $P_{s t}(x+2 \pi n)=P_{s t}(x)$ for all $n$, we have

$$
\begin{aligned}
P_{s t}(x)= & C\left\{P_{W}(x)\left[I-\left(1-e^{-2 \pi b y}\right) \int_{0}^{x} e^{V(z)} d z\right]\right. \\
& \left.-\frac{\Lambda}{2} e^{-V(x)}\left[I_{1}-\left(1-e^{-2 \pi b y}\right) \int_{0}^{x} e^{V(z)}\left(V^{\prime}\right)^{2} d z\right]\right\},
\end{aligned}
$$

where $I=\int_{0}^{2 \pi} e^{V(x)} d x, I_{1}=\int_{0}^{2 \pi}\left[V^{\prime}(x)\right]^{2} e^{V(x)} d x$, and $C$ is the normalizing constant determined by $\int_{0}^{2 \pi} P_{s t}(x) d x=1$. For zero tilt-i.e., $y=0$ - the stationary solution, Eq. (19), reduces to the Wigner distribution in configuration space: viz., $P_{s t}(x)$ $=C P_{W}(x)$. The stationary solution $P_{s t}^{A}(x)$ of Eq. (10) can be found in like manner. In the classical limit, Eq. (19) has been used in Refs. [36,37] in order to calculate the dc currentvoltage characteristics.

However, in spite of the relatively simple quadrature solution, as shown by Risken [15] and Coffey et al. [28], the most efficient method of calculation of both stationary and nonstationary solutions is via continued fractions as they circumvent the problem of evaluating integrals of transcendental functions similar to those encountered in the stationary quadrature solution, Eq. (19). Moreover, continued fractions lend themselves very naturally to computational algorithms. We may implement this method by recalling that, in the stationary state, the periodic distribution function $P_{s t}(x)$ can be expanded in a Fourier series in $x[15]$ : viz.,

$$
P_{s t}(x)=\frac{1}{2 \pi} \sum_{n=-\infty}^{\infty} C_{n} e^{i n x}
$$

where $C_{n}=\left\langle e^{-i n x}\right\rangle_{0}=\int_{0}^{2 \pi} e^{-i n x} P_{s t}(x) d x$ and the angular brackets (with zero subscript) denote the stationary ensemble average. Using this expansion either directly in Eq. (13) or simply by omitting the time derivative and the second-order terms in Eq. (16), we obtain the recurrence relation for the Fourier coefficients $C_{n}$ to terms linear in $\Lambda$ : viz.,

$$
Q_{n} C_{n}+Q_{n}^{+} C_{n+1}+Q_{n}^{-} C_{n-1}=0
$$

where

$$
Q_{n}=-(n / b+i y), \quad Q_{n}^{ \pm}=\mp(1 \pm n \Lambda) / 2 .
$$

Equation (21) can be rearranged as the infinite continued fraction $S_{n}=C_{n} / C_{n-1}$ so that

$$
S_{n}=\frac{Q_{n}^{-}}{-Q_{n}-Q_{n}^{+} S_{n+1}}=\frac{Q_{n}^{-}}{-Q_{n}-\frac{Q_{n}^{+} Q_{n+1}^{-}}{-Q_{n+1}-\frac{Q_{n+1}^{+} Q_{n+2}^{-}}{-Q_{n+2}-\cdots}}} .
$$

Thus, just as the classical case [28], all the $C_{n}$ can simply be calculated via continued fractions as

$$
C_{n}=S_{n} C_{n-1}=S_{n} S_{n-1} \cdots S_{1}
$$

(noting that $C_{0}=1$ ). In particular, we have

$$
C_{1}=S_{1} .
$$

Having determined $C_{n}$, we can calculate the stationary distribution, Eq. (20).

In the classical limit $(\Lambda=0)$, Eqs. (23) and (25) yield the known results [28]

$$
\begin{aligned}
S_{n}^{c l} & =\frac{1 / 2}{n b^{-1}+i y+\frac{1 / 4}{(n+1) b^{-1}+i y+\frac{1 / 4}{(n+2) b^{-1}+i y+\cdots}}} \\
& =\frac{I_{n+i y b}(b)}{I_{n-1+i y b}(b)}
\end{aligned}
$$

and

$$
C_{1}^{c l}=I_{1+i y b}(b) / I_{i y b}(b),
$$

respectively, where $I_{\nu}(z)$ is the modified Bessel function of the first kind of order $\nu$ [54]. The stationary solution of Ankerhold's equation (10) may be determined in like manner. We recall that all such solutions are valid only to terms linear in $\Lambda$. The stationary solution of Eq. (16) to $o\left(\Lambda^{2}\right)$ is given in Appendix A using matrix continued fractions.

\section{MEAN FIRST PASSAGE TIME AND ESCAPE RATES}

The QSE can be used to estimate quantum effects in the various characteristic times of the system (such as the inverse escape rate, mean first passage time, etc.) in the VHD limit. These times are important parameters of the Josephson junction or the ring laser gyroscope as they effectively yield a measure of the phase slip. For simplicity, we consider zero tilt only: i.e., $y=0$. A semiclassical correction to the classical Kramers escape rate $\Gamma_{c l}$ of a Brownian particle over a potential barrier $\Delta V$ in the VHD limit and above the crossover temperature $T_{C}$ (at which the parabolic or inverted harmonic oscillator approximation for the potential is valid near the top of the barrier) can then be written [20]

$$
\Gamma=\Xi \Gamma_{c l} .
$$

Equation (28) constitutes the classical VHD Kramers escape rate $\Gamma_{c l}=\frac{m \omega_{c} \omega_{a}}{2 \pi \zeta} e^{-\beta \Delta V}$ multiplied by Wigner's quantum correction factor derived using TST $[8,9,19]$ : 


$$
\Xi=\frac{\omega_{c}}{\omega_{a}} \frac{\sinh \left(\hbar \beta \omega_{a} / 2\right)}{\sin \left(\hbar \beta \omega_{c} / 2\right)}=1+\frac{\beta^{2} \hbar^{2}}{24}\left(\omega_{c}^{2}+\omega_{a}^{2}\right)+\cdots,
$$

where $\omega_{c}=\sqrt{\left|V^{\prime \prime}\left(x_{c}\right)\right| / m}$ and $\omega_{a}=\sqrt{V^{\prime \prime}\left(x_{a}\right) / m}$ are the barrier and well frequencies [points $c$ and $a$ are, respectively, the maximum and minimum of the potential $V(x)]$. The form of Eq. (28) reinforces our previous contention [20] that we are essentially treating our system at $T>T_{C}$ as a quantum particle embedded in a classical bath, where the diffusion coefficient is modified to take into account quantum dissipative effects due to the bath-particle interactions. The quantum correction terms in Eq. (28) are (as they must be) in complete agreement with Wigner's calculation of the escape rate [21] and in effect reduce the barrier height. The physical origin of the corrections is tunneling at relatively high temperatures near the top of the barrier. In the context of the quantum intermediate- to high-damping (IHD) Kramers rate [20], we remark that the appropriate quantum correction factor was first derived by Wolynes [55] and later by Pollak [56]. The quantum (IHD) correction factor yielded by these calculations is for Ohmic friction [55]

$$
\Xi_{W}=\prod_{n=1}^{\infty} \frac{\omega_{a}{ }^{2}+(2 \pi n / \hbar \beta)^{2}+2 \pi n \gamma / \hbar \beta}{-\omega_{c}{ }^{2}+(2 \pi n / \hbar \beta)^{2}+2 \pi n \gamma / \hbar \beta} .
$$

A comprehensive analysis of Eq. (29) has been made by Grabert et al. [57], Hänggi et al. [58], and also Weiss [8], where it is shown how Wigner's quantum correction $\Xi$ is recovered in the high-temperature limit. In this particular instance the damping independent $\Xi$ is a fair approximation to $\Xi_{W}$ in the VHD limit. This result suggests that replacement of the equilibrium distribution function by that of the closed system may ultimately yield reasonable semiclassical approximations to the actual time-dependent quantum distribution.

The longest relaxation time $\tau_{L}$ can now be estimated as $\tau_{L} \sim \Gamma^{-1}$. However, noting the explicit forms of the diffusion coefficient $D(x)$ and stationary distributions $P_{W}(x)$ and $P_{A}(x)$, one can also estimate the longest relaxation time $\tau_{L}$ using the mean first passage time $\tau_{M F P T}$. For a cosine potential, the mean first passage times for both our QSE (2), and that of Ankerhold et al., QSE (10), may be given by quadratures just as the classical Smoluchowski equation. We have, following the methods described in [15],

$$
\tau_{M F P T}=\int_{0}^{2 \pi} \frac{d x}{P_{W}(x) D(x)} \int_{0}^{x} P_{W}(y) d y
$$

and

$$
\tau_{M F P T}^{A}=\int_{0}^{2 \pi} \frac{d x}{P_{A}(x) D(x)} \int_{0}^{x} P_{A}(y) d y
$$

respectively. In the classical limit $\Lambda \rightarrow 0$, both equations reduce to the classical $\tau_{M F P T}^{c l}$. In Fig. 2, we have plotted the quantum correction factors

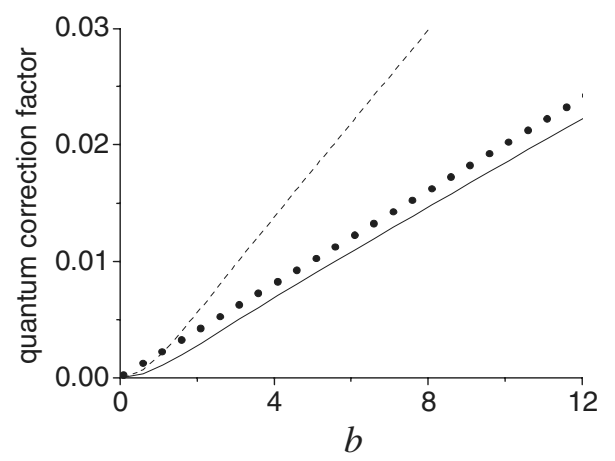

FIG. 2. The quantum correction factors $\Xi-1=\Gamma / \Gamma_{c l}-1=2 b \Lambda$ (solid circles), $\tau_{M F P T}^{c l} / \tau_{M F P T}-1$ (solid line), and $\tau_{M F P T}^{c l} / \tau_{M F P T}^{A}-1$ (dashed line) vs $b$ (Josephson coupling energy) with $\Lambda=0.001$.

$$
\begin{aligned}
\Xi-1= & \Gamma / \Gamma_{c l}-1=2 b \Lambda, \quad \tau_{M F P T}^{c l} / \tau_{M F P T}-1, \\
& \tau_{M F P T}^{c l} / \tau_{M F P T}^{A}-1 .
\end{aligned}
$$

Clearly, the correction factor $\tau_{M F P T}^{c l} / \tau_{M F P T}^{A}-1$ using Eq. (31) derived from the QSE of Ankerhold et al. [43] deviates considerably from the Wigner correction $\Xi-1$ while our QSE predicts the same quantitative behavior of $\tau_{M F P T}^{c l} / \tau_{M F P T}-1$ as the Wigner correction. The discrepancy in the two results is entirely due to the different behavior of the stationary distributions $P_{s t}(x)$ and $P_{s t}^{A}(x)$. Therefore, it appears that Eq. (31) derived from the Ankerhold QSE (10), exaggerates the quantum effects.

\section{DC CURRENT-VOLTAGE CHARACTERISTICS OF A POINT JOSEPHSON JUNCTION}

Now, the tilted cosine potential model may also be used to calculate the dc current-voltage characteristics of a point Josephson junction, which, of course, depend only on the stationary distribution. In order to determine these, we first note that, ignoring the capacitance, the current balance equation for the junction becomes in dimensionless variables [28]

$$
\langle v\rangle_{0}=y-\langle\sin x\rangle_{0}
$$

with $\langle v\rangle_{0}=\langle\dot{x}\rangle_{0}$ denoting the dimensionless average voltage in the stationary state. Equation (32) determines the dc current-voltage characteristic because we can find $\langle\sin x\rangle_{0}$ merely by extracting the imaginary part of $C_{1}$ given by the continued fraction Eq. (25) since

$$
C_{1}=\left\langle e^{-i x}\right\rangle_{0}=\langle\cos x\rangle_{0}-i\langle\sin x\rangle_{0} .
$$

Thus, we have

$$
\langle v\rangle_{0}=y+\operatorname{Im}\left[C_{1}\right] .
$$

We can also calculate from Eq. (33) the normalized differential resistance of the junction: namely,

$$
\frac{d}{d y}\langle v\rangle_{0}=1+\frac{d}{d y} \operatorname{Im}\left[C_{1}\right] .
$$

As shown in Appendix B, the coefficient $C_{1}$ from Eq. (25) can also be expressed in terms of the modified Bessel functions of the first kind, $I_{\nu}(z)$, as the perturbation expansion 

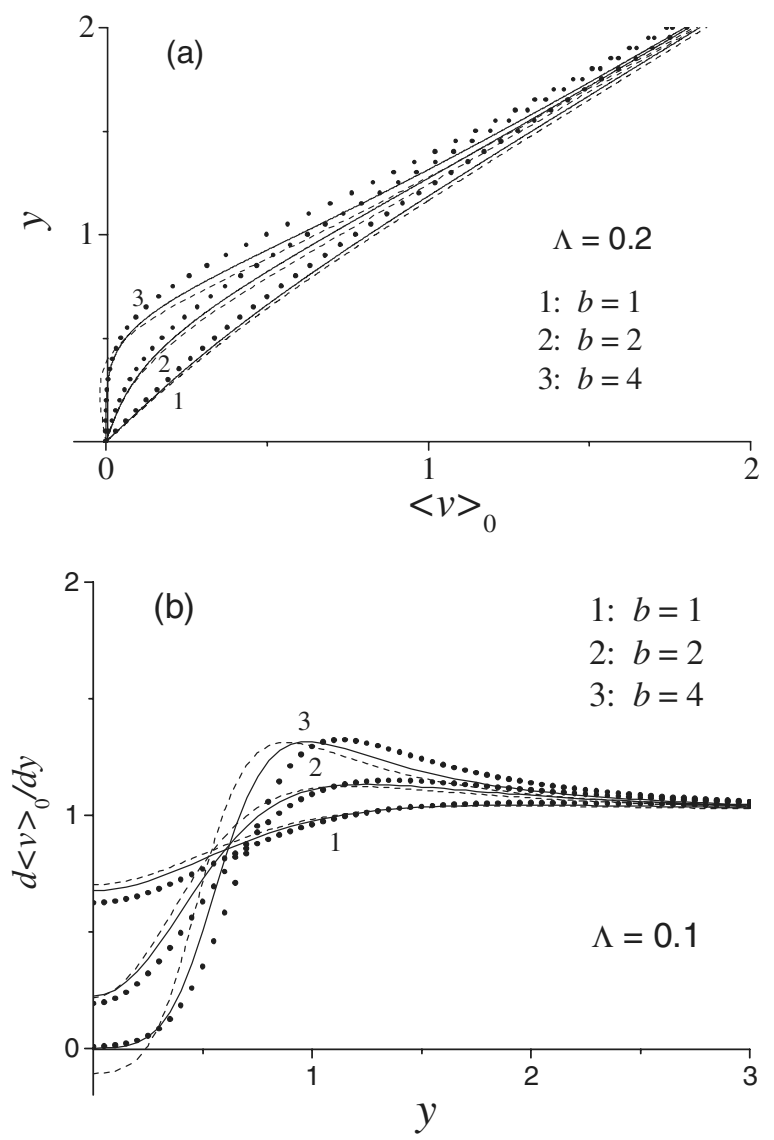

FIG. 3. (a) Quantum effects on the current-voltage [Eq. (33)] and (b) differential resistance-current [Eq. (34)] characteristics of a Josephson junction in the presence of noise for $\Lambda=0.2$ and various values of the Josephson coupling energy $b=1,2$, and 4. Solid and dashed lines are the predictions of Eq. (2) and of the Ankerhold equation (10), respectively. Dots: classical limit $\Lambda=0$.

$$
\begin{aligned}
C_{1}= & C_{1}^{c l}+\Lambda C_{1}^{(1)}+\cdots=\frac{I_{1+i y b}(b)}{I_{i y b}(b)} \\
& +\frac{\Lambda}{I_{i y b}^{2}(b)} \sum_{n=1}^{\infty}\left\{(-1)^{n} n I_{n+i y b}(b)\left[I_{n+1+i y b}(b)+I_{n-1+i y b}(b)\right]\right\} \\
& +\cdots
\end{aligned}
$$

In the classical limit, Eqs. (33) and (34) yield the known results [28] for the characteristics

$$
\langle v\rangle_{0}=y+\operatorname{Im}\left[I_{1+i y b}(b) / I_{i y b}(b)\right]
$$

and

$$
\frac{d}{d y}\langle v\rangle_{0}=1-\operatorname{Re}\left[\frac{2}{I_{i y b}^{2}(b)} \int_{0}^{b} I_{i y b}(z) I_{1+i y b}(z) d z\right] .
$$

The current-voltage characteristic, Eq. (33), and normalized differential resistance, Eq. (34), for both forms of the QSE, Eqs. (2) and (10), calculated using $C_{1}$ to $o(\Lambda)$, rendered by the continued-fraction solution, are shown in Fig. 3 along with the classical results from Eqs. (36) and (37). The quantum effects due to high-temperature nondissipative tunneling near the top of the barrier are readily detectable for large
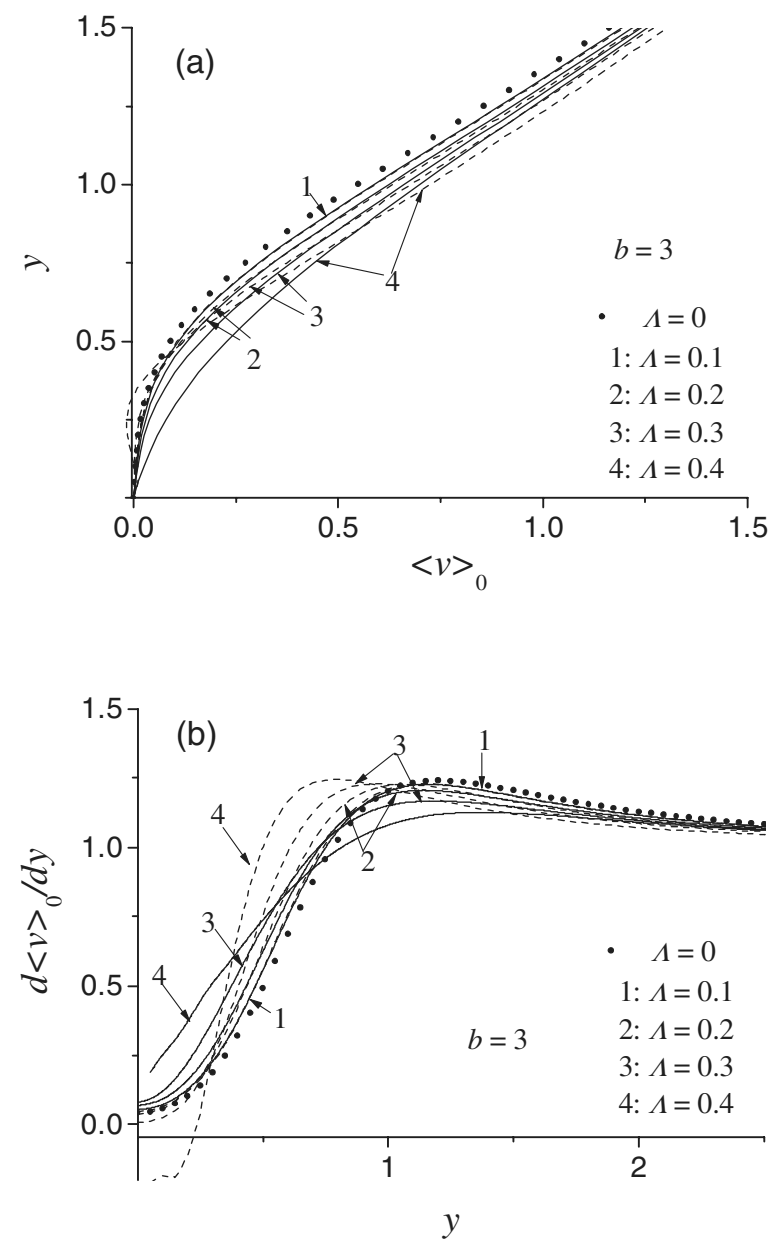

FIG. 4. (a) Quantum effects on the current-voltage, Eq. (33), and (b) differential resistance-current, Eq. (34), characteristics of a Josephson junction in the presence of noise for $b=3$ and various values of the quantum parameter $\Lambda=0$ (dots, classical limit), 0.1 (curves 1), and 0.2 (curves 2), 0.3 (curves 3), and 0.4 (curves 4). Dashed and solid lines: first- and second-order corrections, respectively.

supercurrents and relatively small bias. They comprise an enhanced current for a given voltage, Fig. 3(a), and an enhanced slope of the differential resistance, Fig. 3(b). We note the unphysical behavior of the characteristics calculated from the QSE proposed by Ankerhold et al. [43] for large barrier values $b$ (where the superconducting behavior is pronounced) whereby negative resistance is predicted for zero voltage, Fig. 3(a). This behavior is particularly pronounced in Fig. 3(b) where the continued fraction generated by Eq. (10) predicts negative differential resistance. In all cases the deviation from the classical behavior is most marked for large $b$-i.e., large Josephson coupling energy. In Fig. 4, the convergence of the perturbation procedure is investigated by comparing the first- and second-order perturbation solutions of the QSE (2) obtained by solving Eq. (16) to $o\left(\Lambda^{2}\right)$. Clearly, the first-order perturbation solution closely approximates the second-order one for small $\Lambda$ (here for $\Lambda=0.1$ ). Moreover, the greater $\Lambda$ and $b$ are, the higher the order of perturbation theory required.

Having determined the stationary solution, we now consider the nonstationary problem of the linear impedance of 
the junction for the QSE (13). The impedance can be found using the continued-fraction method or in approximate closed form as two Lorentzians from the effective eigenvalue aftereffect solution just as in the classical case [28].

\section{LINEAR RESPONSE OF THE JOSEPHSON JUNCTION TO AN APPLIED ALTERNATING CURRENT}

In order to evaluate the linear ac response of the Josephson junction, we suppose that the tilt now becomes modulated so that $y \rightarrow y+y_{m} e^{-i \omega t}$, where $b y_{m} \ll 1$, so that the tilt is weakly perturbed (corresponding to a small signal ac superimposed on the dc bias current). We can then make the perturbation expansion

$$
c_{n}=c_{n}^{0}+A_{n}(\omega) y_{m} e^{-i \omega t}+\cdots,
$$

with $A_{0}(\omega)=0$ and $c_{n}^{0}=\prod_{k=1}^{n} S_{k}$ is the unperturbed solution. In particular, simply by evaluating the Fourier amplitude $A_{1}(\omega)$ one may evaluate the linear impedance $Z(\omega)=R_{\omega}-i X_{\omega}$ of the junction (here $R_{\omega}$ and $X_{\omega}$ are the dynamic resistance and the reactance, respectively) by recalling that the averaged current-balance equation in the presence of the ac current is

$$
\langle v\rangle_{0}+\langle v\rangle_{1}=y+y_{m} e^{-i \omega t}-\langle\sin \phi\rangle_{0}-\langle\sin \phi\rangle_{1},
$$

where the subscript " 0 " on the angular brackets denotes the average in the absence of the ac current and the subscript " 1 " the portion of the average which is linear in $y_{m}$. Thus we have

$$
\langle v\rangle_{1}=y_{m} e^{-i \omega t}-\langle\sin \phi\rangle_{1}=Z(\omega) I_{m} e^{-i \omega t} /(R I)
$$

where $Z(\omega)$ is the linear impedance of the junction given by

$$
Z(\omega)=R\left\{1-i\left[A_{1}(\omega)-A_{-1}(\omega)\right] / 2\right\} .
$$

By substituting Eq. (38) into Eq. (16), with $y$ replaced by $y+y_{m} e^{-i \omega t}$, and keeping only terms linear in $y_{m} e^{-i \omega t}$ and $\Lambda$, we obtain the inhomogeneous three-term recurrence relation for the Fourier amplitudes $A_{n}(\omega)$ : viz.,

$$
Q_{n}^{-} A_{n-1}(\omega)+Q_{n}^{+} A_{n+1}(\omega)+\left[Q_{n}+i \omega /(b n)\right] A_{n}(\omega)=i c_{n}^{0} .
$$

The exact solution of the three-term recurrence relation (40) is [22]

$$
A_{1}(\omega)=-i \sum_{n=1}^{\infty} c_{n}^{0} \prod_{k=1}^{n} Q_{k-1}^{+} \Delta_{k}(\omega),
$$

where the continued fraction $\Delta_{k}(\omega)$ is defined by the recurrence equation

$$
\Delta_{k}(\omega)=\left[-i \omega /(b k)-Q_{k}-Q_{k}^{+} Q_{k+1}^{-} \Delta_{k+1}(\omega)\right]^{-1}
$$

Noting that $S_{k}=Q_{k}^{-} \Delta_{k}(0)$ so that $c_{n}^{0}=\prod_{k=1}^{n} Q_{k}^{-} \Delta_{k}(0)$, we can rewrite Eq. (41) as

$$
A_{1}(\omega)=2 i \sum_{n=1}^{\infty} \prod_{k=1}^{n} Q_{k-1}^{+} Q_{k}^{-} \Delta_{k}(0) \Delta_{k}(\omega) .
$$

We remark that $A_{-1}(\omega)$ can be calculated as $A_{-1}(\omega)$ $=A_{1}^{*}(-\omega)$ [28]. Equation (42) combined with Eq. (39) consti- tutes the first-order perturbation solution for the linear ac response in terms of sums of products of continued fractions, allowing one to evaluate the linear impedance of the Josephson junction. When $\omega=0$, Eqs. (39) and (42) yield the differential resistance of the junction, $Z(0) / R=d\langle v\rangle_{0} / d y$, Eq. (34), as they should.

Although Eqs. (39) and (42) are simple as far as numerical computation is concerned, their analytic form is rather cumbersome, rendering a clear physical interpretation of the behavior difficult, so that simplified equations are preferable. These may be obtained by using the effective eigenvalue method just as in the classical case [28]. The method then yields a simple analytic expression for the impedance as we shall now illustrate. By substituting the perturbation expansion $c_{n}(t)=c_{n}^{0}+c_{n}^{1}(t)+\cdots$, where the superscript denotes the power of the applied field into Eq. (16), and replacing $y$ by $y+y_{m} e^{-i \omega t}$, we obtain for $n= \pm 1$

$$
\begin{aligned}
& \frac{d}{d t} c_{ \pm 1}^{1}(t)+(1 \pm i b y) c_{ \pm 1}^{1}(t) \\
& \quad=-\frac{b}{2}(1+\Lambda) c_{ \pm 2}^{1}(t) \mp i b y_{m} c_{ \pm 1}^{0} e^{-i \omega t} .
\end{aligned}
$$

Equation (43) is a differential-recurrence relation for the linear response, which is not closed. Nevertheless, by using the effective eigenvalue method (see for details Ref. [28]), we can rewrite Eq. (43) as two approximate closed ordinary differential equations

$$
\frac{d}{d t} c_{ \pm 1}^{1}(t)+\lambda_{e f}^{ \pm} c_{ \pm 1}^{1}(t)=\mp i b y_{m} c_{ \pm 1}^{0} e^{-i \omega t},
$$

where $\lambda_{e f}^{ \pm}=1 / \tau_{e f}^{( \pm 1)}=\lambda^{\prime} \pm i \lambda^{\prime \prime}$ are a pair of effective eigenvalues and the effective relaxation times $\tau_{e f}^{( \pm 1)}$ are given in terms of continued fractions in Appendix C. The steady-state solution of Eq. (44) is given by the single-pole approximation

$$
c_{ \pm 1}^{1}=\mp \frac{i b S_{ \pm 1}}{\lambda_{e f}^{ \pm}-i \omega} y_{m} e^{-i \omega t},
$$

where $S_{-1}=S_{1}^{*}$ are the equilibrium continued-fraction solutions defined by Eq. (23). Noting Eqs. (39) and (45), we obtain the impedance $Z(\omega)$ in terms of the effective eigenvalues as

$$
\frac{Z(\omega)}{R}=1-\frac{b}{2}\left(\frac{S_{1}}{\lambda_{e f}^{+}-i \omega}+\frac{S_{1}^{*}}{\lambda_{e f}^{+*}-i \omega}\right) .
$$

This expansion merely comprises two Lorentzians, representing damped oscillatory behavior since $\lambda_{e f}^{+}$is complex, and so is much simpler than the continued-fraction solution rendered by Eq. (42). When $\omega=0$, Eq. (46) again yields the correct value of the differential resistance of the junction, $Z(0) / R=d\langle v\rangle_{0} / d y$, Eq. (34). In the classical limit, we can also express the impedance of the junction $Z(\omega)$ in terms of the modified Bessel functions $I_{\nu}(z)$. Using Eq. (26), we have [28] 


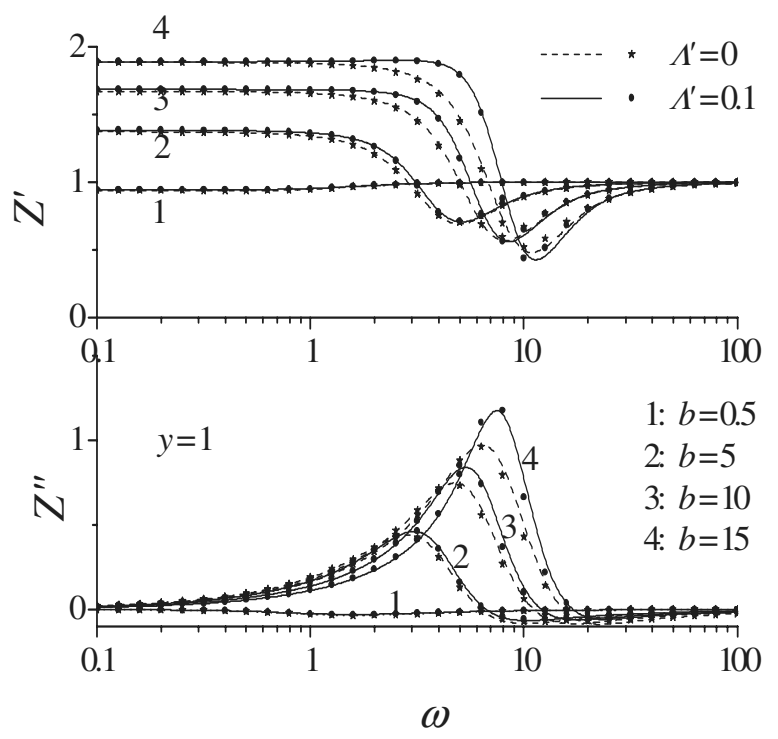

FIG. 5. Quantum effects on the real $\left[Z^{\prime}=\operatorname{Re}(Z)\right]$ and imaginary $\left[Z^{\prime \prime}=\operatorname{Im}(Z)\right]$ parts of the normalized $(R=1)$ impedance vs normalized angular frequency $\omega$ for various values of $b$ (Josephson coupling energy). Comparison of the quantum [solid lines, $\Lambda=0.1$, Eqs. (39) and (42)], classical [dashed lines, $\Lambda=0$, Eqs. (39) and (42)], and approximate [stars, Eq. (46), and solid circles, Eq. (47)] solutions.

$$
\frac{Z(\omega)}{R}=1-\frac{b}{2}\left[\frac{I_{1+i y b}(b)}{I_{i y b}(b)\left(\lambda_{e f}^{+}-i \omega\right)}+\frac{I_{1-i y b}(b)}{I_{-i y b}(b)\left(\lambda_{e f}^{+*}-i \omega\right)}\right],
$$

where $\lambda_{e f}^{+}$is determined by Eq. (C9) of Appendix C.

We now compare the impedance $Z(\omega)$ calculated from the approximate Eqs. (46) and (47) with the continued-fraction solution [Eqs. (39) and (42)]. The results are shown in Figs. 5 and 6 . It is apparent by inspection that the simple effective

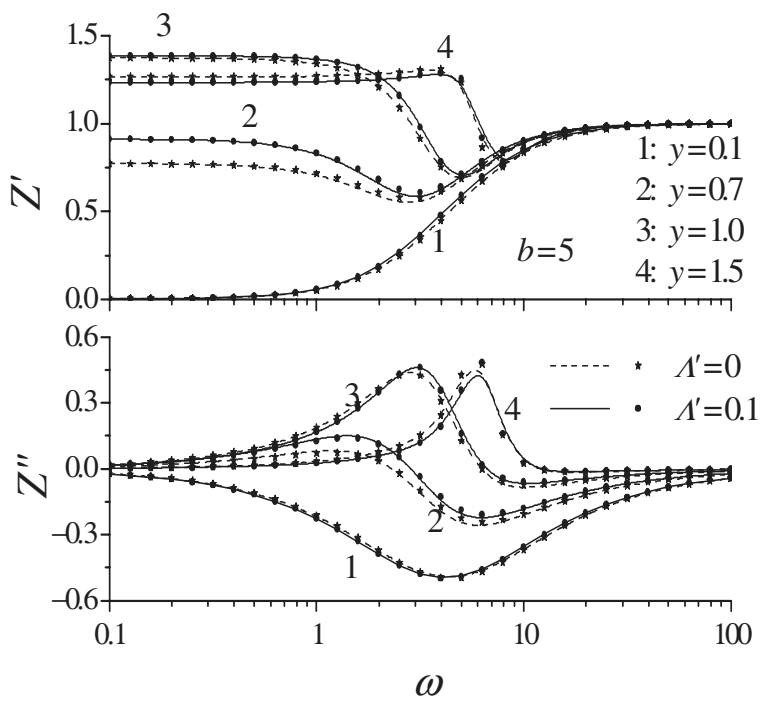

FIG. 6. Quantum effects on the real and imaginary parts of the normalized $(R=1)$ impedance vs $\omega$ for various values of the tilt parameter $y=0.1,0.7,1.0$, and 1.5 and $b=5$. Key as in Fig. 5 .

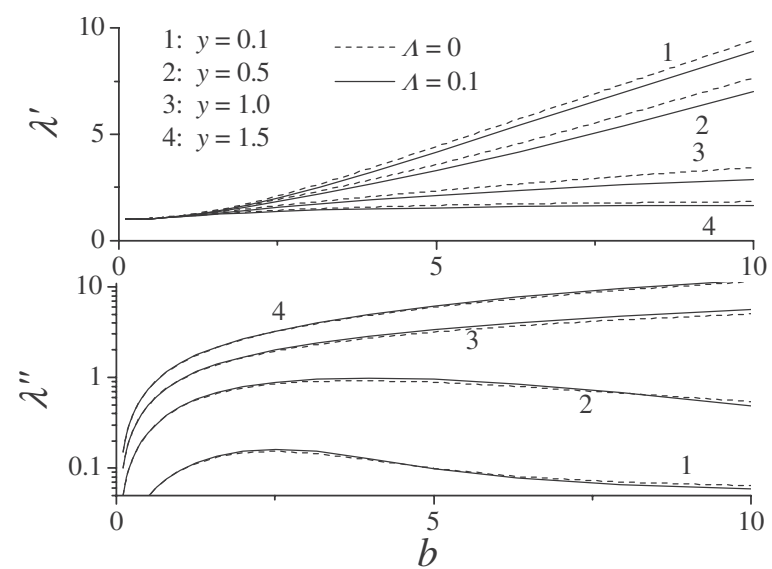

FIG. 7. Quantum effects on the real and imaginary parts of the effective eigenvalue $\lambda_{e f}^{+}=\lambda^{\prime}+i \lambda^{\prime \prime}$ vs $b$ for various values of $y=0.1$, $0.5,1.0$, and 1.5. Solid and dashed lines: Eq. (30) for $\Lambda=0.1$ and $\Lambda=0$ (classical limit), respectively.

eigenvalue solution corresponds almost perfectly to the continued-fraction solution for a wide range of the parameters $y$ and $b$ in both the classical and quantum cases. Moreover, it allows one to represent the impedance of the junction $Z(\omega)$ by the simple analytic formula of Eq. (46), which merely constitutes a damped resonance with natural angular frequency $\lambda^{\prime \prime}$ and damping constant $\lambda^{\prime}$ taking into account the effects of macroscopic tunneling near the top of the barrier. We also remark that the deviations from the classical solution, Eq. (47), predicted by the QSE are significant. The deviations are exemplified in Fig. 5, where for large $b$, corresponding to a large Josephson coupling energy, the resonant peak is considerably enhanced (i.e., we have a higher $Q$ factor) in comparison to the classical one. The reduction in the damping is particularly marked for large barriers and moderate tilts. This is corroborated by Figs. 7 and 8, where the real part of the effective eigenvalue is reduced due to the dissipative tunneling near the top of the barrier. This phenomenon represents a decrease in the damping factor of the Josephson oscillations due to dissipative tunneling, thus enhancing the resonant peak. However, the imaginary part of

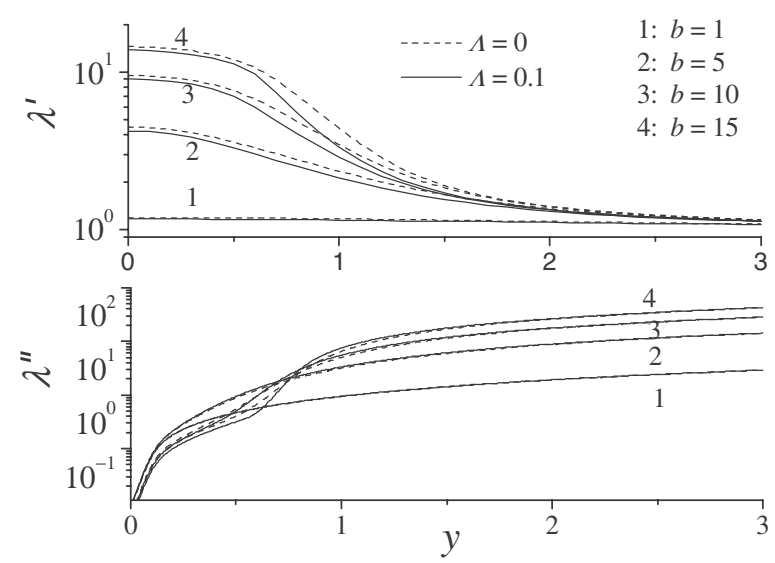

FIG. 8. Quantum effects on the real and imaginary parts of the effective eigenvalue vs $y$ for various values of $b=1,5,10$, and 15 . Key as in Fig. 7. 
the effective eigenvalue or the resonant frequency remains substantially unaltered, again supporting the above conclusion. The nature of the ac response suggests that measurements of this response based on the behavior of the $Q$ factor may constitute a useful estimate of the role played by dissipative macroscopic quantum tunneling in a Josephson junction. The situation should be mirrored in measurements [59] of ferromagnetic resonance in the dynamics of the magnetization of fine single-domain ferromagnetic particles insofar as such experiments should also yield information concerning macroscopic tunneling in these systems.

\section{CONCLUDING REMARKS}

We have demonstrated how the QSE which we have proposed may be used to calculate quantum corrections to the various characteristics of the Josephson tunnelling junction in the zero-capacitance and high-temperature limits. One of the most useful conclusions is that for small values of the quantum parameter all the characteristics may be calculated using a three-term scalar recurrence relation essentially similar to that encountered in the classical solution. Thus in the first order of perturbation theory one may obtain analytic formulas very similar to those of the classical case. The quantum deviations from the classical result so produced are, however, detectable (cf. Fig. 5). We have also illustrated the convergence of the perturbation method by comparing the results for the dc characteristics in first and second order of perturbation theory. Thus it appears that the first-order approximation, which is relatively easy to compute, is valid for small values of the quantum parameter. We have also compared our results with those yielded by another form of the QSE (in which the drift, as well as the diffusion coefficient, is altered), which has been proposed by Ankerhold et al. [43] and subsequently used by other authors [42,45-47]. This equation, however, appears to predict unphysical results such as a negative resistance for zero voltage and negative differential resistance. Moreover, the equilibrium solution of this equation does not reduce to the Wigner configuration-space equilibrium distribution. This appears to be a consequence of neglecting the contribution of the $p^{2}$ term in the Wigner phase-space distribution to the configuration-space distribution as we have already discussed in Ref. [19]. We remark that in a later publication the form of the QSE proposed by us has been accepted by Ankerhold [60] [his Eq. (6.121), p. 161], which is identical to Eq. (2).

We finally remark that the treatment of the tilted cosine potential outlined here may be extended to the general inertial or nonzero-capacitance case. In the classical limit, this has already been accomplished in Ref. [61], where the governing Klein-Kramers equation in phase space giving rise to Brinkman's hierarchy of partial differential recurrence relations $[19,27]$ in configuration space (which are now obtained rather than the Smoluchowski equation) has been solved using matrix continued fractions yielding the escape rate and dynamic structure factor. Moreover, the results agree with Melnikov's [62] asymptotic expression for the escape rate for all values of the damping. These continued-fraction calculations have been extended [17] to the quantum case using
Eq. (1) for zero tilt. The escape rate so calculated again agrees with Melnikov's asymptotic expression for the quantum escape rate $[62,63]$. Thus it is clearly evident that with certain minor modifications, all these calculations could be applied to the evaluation of quantum effects in the characteristics of the Josephson junction including the capacitance. This of course corresponds to a solution valid for all damping ranges since the capacitance of the junction plays the role of inertia in the mechanical analog.

\section{ACKNOWLEDGMENTS}

The Visiting Professors' and Lecturers' fund of Trinity College Dublin is thanked for financial support for S.V.T. in this work. L. C. acknowledges, with thanks, IRCSET.

\section{APPENDIX A: STATIONARY MATRIX CONTINUED-FRACTION SOLUTION OF Eq. (16)}

In the stationary state, we have from Eq. (16)

$$
Q_{n}^{\prime} C_{n}+Q_{n}^{\prime-} C_{n-1}+Q_{n}^{\prime+} C_{n+1}+Q_{n}^{\prime \prime}\left(C_{n-2}+C_{n+2}\right)=0,
$$

where $\quad Q_{n}^{\prime}=-\left(n b^{-1}+i y+n b \Lambda^{2} / 5\right)$, $Q_{n}^{\prime \pm}$ $=\mp\left[1 \pm n \Lambda \mp 3 n \Lambda^{2}(1 \pm i b y) / 5\right] / 2$, and $Q_{n}^{\prime \prime}=b n \Lambda^{2} / 5$. In order to solve Eq. (A1), we introduce the column vectors

$$
\mathbf{C}_{ \pm n}=\left(\begin{array}{c}
C_{ \pm 2 n} \\
C_{ \pm(2 n-1)}
\end{array}\right), \quad \mathbf{C}_{n}=\mathbf{C}_{-n}^{*},
$$

and the single-element initial vector $\mathbf{C}_{0}=(1)$. Noting that $\mathbf{C}_{-1}=\mathbf{C}_{1}^{*}$, we rewrite Eq. (A1) as the set of matrix three-term recurrence equations

$$
\begin{gathered}
\mathbf{Q}_{1}^{-} \mathbf{C}_{0}+\mathbf{Q}_{1} \mathbf{C}_{1}+\mathbf{Q}_{1}^{+} \mathbf{C}_{2}=-\mathbf{F} \mathbf{C}_{1}^{*}, \\
\mathbf{Q}_{n}^{-} \mathbf{C}_{n-1}+\mathbf{Q}_{n} \mathbf{C}_{n}+\mathbf{Q}_{n}^{+} \mathbf{C}_{n+1}=\mathbf{0},
\end{gathered}
$$

for $n=1$ and $n>1$, respectively, where the matrices $\mathbf{Q}_{n}$ and $\mathbf{Q}_{n}^{ \pm}$are given by

$$
\begin{gathered}
\mathbf{Q}_{n}=\left(\begin{array}{cc}
Q_{2 n}^{\prime} & Q_{2 n}^{\prime-} \\
Q_{2 n-1}^{\prime+} & Q_{2 n-1}^{\prime}
\end{array}\right), \quad \mathbf{Q}_{n}^{+}=\left(\begin{array}{cc}
Q_{2 n}^{\prime \prime} & Q_{2 n}^{\prime+} \\
0 & Q_{2 n-1}^{\prime \prime}
\end{array}\right), \\
\mathbf{Q}_{n}^{-}=\left(\begin{array}{cc}
Q_{2 n}^{\prime \prime} & 0 \\
Q_{2 n-1}^{\prime-} & Q_{2 n-1}^{\prime \prime}
\end{array}\right)
\end{gathered}
$$

with $\mathbf{Q}_{1}^{-}$and $\mathbf{F}$ defined as

$$
\mathbf{Q}_{1}^{-}=\left(\begin{array}{c}
Q_{2}^{\prime \prime} \\
Q_{1}^{\prime-}
\end{array}\right), \quad \mathbf{F}=\left(\begin{array}{cc}
0 & 0 \\
0 & Q_{1}^{\prime \prime}
\end{array}\right) .
$$

Thus the vectors $\mathbf{C}_{n}$ can be calculated via matrix continued fractions as [28]

$$
\begin{aligned}
& \mathbf{C}_{1}=\Delta_{1}\left(\mathbf{Q}_{1}^{-} \mathbf{C}_{0}+\mathbf{F} \mathbf{C}_{1}^{*}\right), \\
& \mathbf{C}_{n}=\Delta_{n} \mathbf{Q}_{n}^{-} \cdots \Delta_{2} \mathbf{Q}_{2}^{-} \mathbf{C}_{1},
\end{aligned}
$$

where $\boldsymbol{\Delta}_{n}$ are the matrix continued fractions defined by the recurrence equation 


$$
\boldsymbol{\Delta}_{n}=\left(-\mathbf{Q}_{n}-\mathbf{Q}_{n}^{+} \boldsymbol{\Delta}_{n+1} \mathbf{Q}_{n+1}^{-}\right)^{-1} .
$$

We represent the complex vectors and matrices in Eq. (A2) as $\mathbf{C}_{1}=\mathbf{C}_{1}^{\prime}+i \mathbf{C}_{1}^{\prime \prime}, \boldsymbol{\Delta}_{1} \mathbf{Q}_{1}^{-}=\mathbf{S}_{1}^{\prime}+i \mathbf{S}_{1}^{\prime \prime}$, and $\boldsymbol{\Delta}_{1} \mathbf{F}=\mathbf{f}+i \mathbf{g}$. Equating the real and imaginary parts of Eq. (A2), we obtain simultaneous equations for the unknowns $\mathbf{C}_{1}^{\prime}$ and $\mathbf{C}_{1}^{\prime \prime}$ : namely,

$$
\begin{aligned}
& (\mathbf{I}-\mathbf{f}) \mathbf{C}_{1}^{\prime}-\mathbf{g} \mathbf{C}^{\prime \prime}=\mathbf{S}_{1}^{\prime} \mathbf{C}_{0}, \\
& (\mathbf{I}+\mathbf{f}) \mathbf{C}^{\prime \prime}-\mathbf{g C}_{1}^{\prime}=\mathbf{S}_{1}^{\prime \prime} \mathbf{C}_{0},
\end{aligned}
$$

where $\mathbf{I}$ is the unit matrix. On solving Eqs. (A4) and (A5) for $\mathbf{C}_{1}^{\prime}$ and $\mathbf{C}_{1}^{\prime \prime}$, we find

$$
\begin{aligned}
& \mathbf{C}_{1}^{\prime}=\left[\mathbf{I}-\mathbf{f}-\mathbf{g}(\mathbf{I}+\mathbf{f})^{-1} \mathbf{g}\right]^{-1}\left[\mathbf{S}_{1}^{\prime}+\mathbf{g}(\mathbf{I}+\mathbf{f})^{-1} \mathbf{S}_{1}^{\prime \prime}\right] \mathbf{C}_{0}, \\
& \mathbf{C}_{1}^{\prime \prime}=\left[\mathbf{I}+\mathbf{f}-\mathbf{g}(\mathbf{I}-\mathbf{f})^{-1} \mathbf{g}\right]^{-1}\left[\mathbf{S}_{1}^{\prime \prime}+\mathbf{g}(\mathbf{I}-\mathbf{f})^{-1} \mathbf{S}_{1}^{\prime}\right] \mathbf{C}_{0} .
\end{aligned}
$$

The dc characteristics determined from Eqs. (33), (34), (A6), and (A7) are plotted in Figs. 4(a) and 4(b). The matrix continued-fraction algorithm presented can be applied to the calculation of the second-order correction in the linear impedance of the junction. Furthermore, it can be adapted to any desired order of $\Lambda$.

\section{APPENDIX B: PROOF OF Eq. (35)}

We seek a perturbation solution of Eq. (21) as $C_{n}=C_{n}^{c l}$ $+\Lambda C_{n}^{(1)}$. The recurrence equations for $C_{n}^{c l}$ and $C_{n}^{(1)}$ become

$$
\begin{gathered}
Q_{n} C_{n}^{c l}+Q_{n}^{+} C_{n+1}^{c l}+Q_{n}^{-} C_{n-1}^{c l}=0, \\
Q_{n} C_{n}^{(1)}+Q_{n}^{+} C_{n+1}^{(1)}+Q_{n}^{-} C_{n-1}^{(1)}=n\left(C_{n+1}^{c l}+C_{n-1}^{c l}\right) / 2,
\end{gathered}
$$

where $Q_{n}$ and $Q_{n}^{ \pm}$are defined by Eq. (22). The solution of the homogeneous recurrence equation (B1) is given by [28]

$$
C_{n}^{c l}=S_{n}^{c l} S_{n-1}^{c l} \cdots S_{1}^{c l}=I_{n+i y b}(b) / I_{i y b}(b),
$$

where $S_{n}^{c l}=C_{n}^{c l} / C_{n-1}^{c l}=I_{n+i y b}(b) / I_{n-1+i y b}(b)$. This is easily proved by comparing the continued fraction $S_{n}^{c l}$, viz.,

$$
S_{n}^{c l}=\frac{1 / 2}{n / b+i y+S_{n+1}^{c l} / 2},
$$

with the corresponding continued fraction for the modified Bessel functions $I_{\nu}(z)$, viz. [28],

$$
\frac{I_{\nu}(z)}{I_{\nu-1}(z)}=\frac{1 / 2}{\nu / z+I_{\nu+1}(z) /\left[2 I_{\nu}(z)\right]},
$$

which follows from the underlying recurrence relation for $I_{\nu}(z)$, viz, $I_{\nu-1}(z)-I_{\nu+1}(z)=(2 v / z) I_{\nu}(z)$ [54]. By inspection, the continued fraction $S_{n}^{c l}$ given by Eq. (B3) is identical to Eq. (B4) if $\nu=n+i y b$ and $z=b$. The solution of the inhomogeneous recurrence equation (B2) for $C_{1}^{(1)}$ can be obtained by the standard methods described in [28] and is given by

$$
\begin{aligned}
C_{1}^{(1)} & =\sum_{n=1}^{\infty}(-1)^{n} n\left(C_{n+1}^{c l}+C_{n-1}^{c l}\right) \prod_{k=1}^{n} S_{k}^{c l} \\
& =\frac{1}{I_{i y b}^{2}(b)} \sum_{n=1}^{\infty}(-1)^{n} n I_{n+i y b}(b)\left[I_{n+1+i y b}(b)+I_{n-1+i y b}(b)\right] .
\end{aligned}
$$

Thus we obtain Eq. (35).

\section{APPENDIX C: THE EFFECTIVE RELAXATION TIME AND AFTEREFFECT SOLUTION}

Suppose that at a time $t=-\infty$ the tilt $y$ is incremented by a small value $\Delta$, where $b \Delta \ll 1$. This increment vanishes at $t$ $=0$. The relaxation functions $f_{n}(t)=c_{n}(t)-c_{n}(\infty)$ or aftereffect solutions then obey the differential-recurrence relation

$$
\begin{aligned}
\frac{d}{d t} f_{n}(t)+\left(n^{2}+\text { inby }\right) f_{n}(t)= & \frac{b n}{2}\left[(1-n \Lambda) f_{n-1}(t)\right. \\
& \left.-(1+n \Lambda) f_{n+1}(t)\right] .
\end{aligned}
$$

Equation (C1) is valid only for $t>0$. Now we could solve Eq. (C1) exactly for the linear response, which in general comprises an infinite number of closely spaced exponential relaxation modes, using continued fractions: viz., $f_{n}(t)$ $=\Sigma_{k} a_{k}^{n} e^{-t / \tau_{k}^{(n)}}$. However, the effective relaxation time method [28], whereby the multimodal response is represented by a single mode $f_{n}(t) \approx f_{n}(0) e^{-t / \tau_{e f}^{(n)}}$, which is complex, meaning that the effective relaxation functions display damped oscillatory behavior, yields a close approximation to the exact solution at all times. In order to apply this method, it is supposed that, taking $n=1$ for example,

$$
f_{1}(t)=f_{1}(0) e^{-t / \tau_{e f}^{(1)}} .
$$

Thus the normalized effective relaxation time $\tau_{e f}^{(1)}$ is given by

$$
\tau_{e f}^{(1)}=-f_{1}(t) /\left.\dot{f}_{1}(t)\right|_{t=0} .
$$

An explicit evolution equation for $f_{1}(t)$ can be obtained from Eq. (C1); we have

$$
\frac{d}{d t} f_{1}(t)+(1+i b y) f_{1}(t)=-\frac{b}{2}(1+\Lambda) f_{2}(t),
$$

where we have noted that $f_{0}(t)=0$. Equations (C2) and (C3) thus imply that the effective relaxation time is

$$
\tau_{e f}^{(1)}=\left[1+i b y+b(1+\Lambda) \frac{f_{2}(0)}{2 f_{1}(0)}\right]^{-1} .
$$

Here $f_{n}(0)=c_{n}(0)-c_{n}(\infty)$ at $n=1,2$ can be evaluated in terms of continued fractions since the initial values $c_{n}(0)$ satisfy the recurrence relation

$$
\left(Q_{n}-i b \Delta\right) c_{n}(0)+Q_{n}^{+} c_{n+1}(0)+Q_{n}^{-} c_{n-1}(0)=0,
$$

while the final values $c_{n}(\infty)$ also satisfy Eq. (C5) with $\Delta=0$. Furthermore, as far as the linear response of the junction is concerned, we are only interested in terms linear in $\Delta$. Thus we may express the initial values $c_{n}(0)$ as power series in the perturbation $\Delta$ : viz.,

$$
c_{n}(0)=c_{n}(\infty)+\Delta \partial_{y} c_{n}(0)+o(\Delta) .
$$

The derivatives $\partial_{y} c_{n}(0)$ must now be evaluated, which is done as follows. On substituting Eq. (C6) into Eq. (C5) and noting that $c_{n}(\infty)$ satisfies $Q_{n} c_{n}(\infty)+Q_{n}^{+} c_{n+1}(\infty)+Q_{n}^{-} c_{n-1}(\infty)$ $=0$, we have the recurrence relations for $\partial_{y} c_{n}(0)$ as

$$
Q_{n} \partial_{y} c_{n}(0)+Q_{n}^{-} \partial_{y} c_{n-1}(0)+Q_{n}^{+} \partial_{y} c_{n+1}(0)=i c_{n}(\infty),
$$


where $c_{n}(\infty)$ can be expressed [noting Eq. (24)] in terms of products of continued fractions as

$$
c_{n}(\infty)=S_{n} S_{n-1} \cdots S_{1} .
$$

The solution of the inhomogeneous recurrence Eq. (C7) for $\partial_{y} c_{1}(0)$ can be obtained by standard methods and is given by [noting Eq. (C8)]

$$
\partial_{y} c_{1}(0)=2 i \sum_{n=1}^{\infty} \prod_{k=1}^{n}\left(Q_{k-1}^{+} / Q_{k}^{-}\right) S_{k}^{2} \text {. }
$$

Since $\partial_{y} c_{2}(0)$ can be obtained from Eq. (C7) for $n=1$ as $\partial_{y} c_{2}(0)=\left[i c_{1}(\infty)-Q_{1} \partial_{y} c_{1}(0)\right] / Q_{1}^{+}$, the effective relaxation time from Eq. (C4) is then

$$
\begin{aligned}
\tau_{e f}^{(1)} & =\left[1+i b y+\frac{b}{2}(1+\Lambda) \frac{\partial_{y} c_{2}(0)}{\partial_{y} c_{1}(0)}\right]^{-1}=i \frac{\partial_{y} c_{1}(0)}{b c_{1}(\infty)} \\
& =-\frac{2}{b S_{1}} \sum_{n=1}^{\infty} \prod_{k=1}^{n}\left(Q_{k-1}^{+} / Q_{k}^{-}\right) S_{k}^{2} .
\end{aligned}
$$

The effective relaxation time $\tau_{e f}^{(-1)}$ for $f_{-1}(t)$ is related to $\tau_{e f}^{(1)}$ by $\tau_{e f}^{(-1)}=\tau_{e f}^{(1) *}$.

The behavior of the real and imaginary parts of the inverse of the effective relaxation time $\lambda_{e f}^{+}=1 / \tau_{e f}^{(1)}=\lambda^{\prime}+i \lambda^{\prime \prime}$ as a function of the barrier height $b$ and bias parameter $y$ is illustrated in Figs. 7 and 8. In the classical limit, $\lambda_{e f}^{+}$can be given in terms of modified Bessel functions of the first kind as

$$
\left.\lambda_{e f}^{+}\right|_{\Lambda=0}=\frac{b I_{i y b}(b) I_{1+i y b}(b)}{2 \int_{0}^{b} I_{i y b}(z) I_{1+i y b}(z) d z} .
$$

The deviations from the classical equation (C9) are appreciable as illustrated by Figs. 7 and 8 .
[1] E. P. Wigner, Phys. Rev. 40, 749 (1932).

[2] J. E. Moyal, Proc. Cambridge Philos. Soc. 45, 99 (1949).

[3] S. R. de Groot and L. G. Suttorp, Foundations of Electrodynamics (North-Holland, Amsterdam, 1972).

[4] M. Hillery, R. F. O'Connell, M. O. Scully, and E. P. Wigner, Phys. Rep. 106, 121 (1984).

[5] H. W. Lee, Phys. Rep. 259, 147 (1995).

[6] W. P. Schleich, Quantum Optics in Phase Space (Wiley-VCH, Berlin, 2001).

[7] Quantum Mechanics in Phase Space, edited by C. K. Zachos, D. B. Fairlie, and T. L. Curtright (World Scientific, Singapore, 2005).

[8] U. Weiss, Quantum Dissipative Systems, 3rd ed. (World Scientific, Singapore, 2008).

[9] G. S. Agarwal, Phys. Rev. A 4, 739 (1971).

[10] A. O. Caldeira and A. J. Leggett, Physica B \& C 121, 587 (1983).

[11] Y. Tanimura and P. G. Wolynes, Phys. Rev. A 43, 4131 (1991); J. Chem. Phys. 96, 8485 (1992).

[12] J. J. Halliwell and T. Yu, Phys. Rev. D 53, 2012 (1996).

[13] H. Grabert, Chem. Phys. 322, 160 (2006).

[14] R. Kapral, Annu. Rev. Phys. Chem. 57, 129 (2006).

[15] H. Risken, The Fokker-Planck Equation, 2nd ed. (SpringerVerlag, Berlin, 1989).

[16] J. L. García-Palacios, Europhys. Lett. 65, 735 (2004); J. L. García-Palacios and D. Zueco, J. Phys. A 37, 10735 (2004).

[17] W. T. Coffey, Yu. P. Kalmykov, S. V. Titov, and B. P. Mulligan, Europhys. Lett. 77, 20011 (2007); Phys. Rev. E 75, 041117 (2007).

[18] W. T. Coffey, Yu. P. Kalmykov, and S. V. Titov, J. Chem. Phys. 127, 074502 (2007).

[19] W. T. Coffey, Yu. P. Kalmykov, S. V. Titov, and B. P. Mulligan, J. Phys. A 40, F91 (2007).

[20] W. T. Coffey, Yu. P. Kalmykov, S. V. Titov, and B. P. Mulli- gan, Phys. Chem. Chem. Phys. 9, 3361 (2007).

[21] E. P. Wigner, Z. Phys. Chem. Abt. B 19, 203 (1932).

[22] H. Grabert, P. Schramm, and G. L. Ingold, Phys. Rep. 168, 115 (1988).

[23] Yu. P. Kalmykov, W. T. Coffey, and S. V. Titov, Phys. Rev. E 76, 051104 (2007); Phys. Rev. B 77, 104418 (2008); J. Phys. A 41, 105302 (2008).

[24] R. L. Stratonovich, Zh. Eksp. Teor. Fiz. 31, 1012 (1956); [Sov. Phys. JETP 4, 891 (1957)].

[25] H. A. Kramers, Physica (Amsterdam) 7, 284 (1940).

[26] S. Chandrasekhar, Rev. Mod. Phys. 15, 1 (1943).

[27] H. C. Brinkman, Physica (Amsterdam) 22, 29 (1956).

[28] W. T. Coffey, Yu. P. Kalmykov, and J. T. Waldron, The Langevin Equation, 2nd ed. (World Scientific, Singapore, 2004).

[29] H. Weyl, Z. Phys. 46, 1 (1927).

[30] G. Barone and A. Paterno, Physics and Applications of the Josephson Effect (Wiley, New York, 1982).

[31] K. K. Likharev, Dynamics of Josephson Junctions and Circuits (Gordon and Breach, New York, 1986).

[32] M. Tinkham, Introduction to Superconductivity, 2nd ed. (McGraw-Hill, New York, 1996).

[33] W. W. Chow, J. Gea-Banacloche, L. M. Pedrotti, V. Sanders, W. Schleich, and M. O. Scully, Rev. Mod. Phys. 57, 61 (1985)

[34] S. G. Chung, Phys. Rev. B 29, 6977 (1984).

[35] L. Machura, M. Kostur, P. Hänggi, P. Talkner, and J. Łuczka, Phys. Rev. E 70, 031107 (2004).

[36] V. Ambegaokar and B. I. Halperin, Phys. Rev. Lett. 22, 1364 (1969)

[37] Yu. M. Ivanchenko and L. A. Zil'berman, Zh. Eksp. Teor. Fiz. 55, 2395 (1968); [Sov. Phys. JETP 28, 1272 (1969)].

[38] Single Charge Tunneling, edited by H. Grabert and M. H. Devoret (Plenum Press, New York, 1992).

[39] M. H. Devoret, D. Esteve, C. Urbina, J. Martinis, A. Cleland, 
and J. Clarke, in Quantum Tunneling in Solids, edited by Yu. Kagan and A. J. Leggett (Elsevier, Amsterdam, 1992).

[40] G. L. Ingold and H. Grabert, Phys. Rev. Lett. 83, 3721 (1999); H. Grabert, G. L. Ingold, and B. Paul, Europhys. Lett. 44, 360 (1998).

[41] J. Ankerhold, Europhys. Lett. 67, 280 (2004).

[42] L. Machura, M. Kostur, P. Talkner, J. Łuczka, and P. Hänggi, Phys. Rev. E 73, 031105 (2006).

[43] J. Ankerhold, P. Pechukas, and H. Grabert, Phys. Rev. Lett. 87, 086802 (2001); Chaos 15, 026106 (2005).

[44] J. Ankerhold, Phys. Rev. E 64, 060102(R) (2001).

[45] J. Łuczka, R. Rudnici, and P. Hänggi, Physica A 351, 60 (2005).

[46] L. Machura, J. Łuczka, P. Talkner, and P. Hänggi, Acta Phys. Pol. B 38, 1855 (2007).

[47] J. Dajka, L. Machura, Sz. Rogoziński, and J. Łuczka, Phys. Rev. B 76, 045337 (2007).

[48] H. Grabert, Chem. Phys. 322, 160 (2006).

[49] E. P. Gross and J. L. Lebowitz, Phys. Rev. 104, 1528 (1956).

[50] A. G. Redfield, IBM J. Res. Dev. 1, 19 (1957).

[51] E. Geva, E. Rosenman, and D. Tannor, J. Chem. Phys. 113, 1380 (2000).
[52] J. T. Anderson and A. M. Goldman, Phys. Rev. Lett. 23, 128 (1969).

[53] C. M. Falco, W. H. Parker, S. E. Trullinger, and P. K. Hansma, Phys. Rev. B 10, 1865 (1974).

[54] Handbook of Mathematical Functions, edited by M. Abramowitz and I. Stegun (Dover, New York, 1964).

[55] P. G. Wolynes, Phys. Rev. Lett. 47, 968 (1981).

[56] E. Pollak, Chem. Phys. Lett. 127, 178 (1986); J. Chem. Phys. 85, 865 (1986).

[57] H. Grabert, P. Olschowski, and U. Weiss, Phys. Rev. B 36, 1931 (1987).

[58] P. Hänggi, H. Grabert, G. L. Ingold, and U. Weiss, Phys. Rev. Lett. 55, 761 (1985).

[59] Yu. P. Kalmykov and W. T. Coffey, Phys. Rev. B 56, 3325 (1997).

[60] J. Ankerhold, Quantum Tunnelling in Complex Systems (Springer-Verlag, Berlin, 2007).

[61] W. T. Coffey, Yu. P. Kalmykov, S. V. Titov, and B. P. Mulligan, Phys. Rev. E 73, 061101 (2006).

[62] V. I. Mel'nikov, Physica A 130, 606 (1985); Phys. Rep. 209, 1 (1991).

[63] I. Rips and E. Pollak, Phys. Rev. A 41, 5366 (1990). 